\title{
Estudio Cadavérico del Arco de la Vena Cefálica
}

\author{
Cadaveric Study of the Cephalic Vein Arch
}

\author{
Alejandra Masilla; Sofía Mansilla; Andrés Pouy; Alejandra Garretano; \\ Juan Ignacio Améndola; Alejandro Russo \& Eduardo Olivera
}

\begin{abstract}
MANSIlla, A.; MANSIlla, S.; POUY, A.; GARRETANO, A.; AMÉNDOLA, J. I.; RUSSO, A. \& OLIVERA, E. Estudio cadavérico del arco de la vena cefálica. Int. J. Morphol., 38(5):1229-1234, 2020.

RESUMEN: Dentro de las disfunciones de los angioaccesos, la estenosis del arco de la vena cefálica (AVC) juega un papel significativo. Existen características anatómicas del AVC que explican, en parte, este fenómeno. Todo esto obliga a un conocimiento profundo del AVC y sus principales variantes. Se disecaron $43(\mathrm{n}=43)$ axilas de cadáveres adultos fijados en solución en base a formol. Se registró: longitud del AVC (LAC), angulación del AVC respecto a la vena cefálica (AAC), tipo de terminación (simple o múltiple), presencia de afluentes y su número. LAC promedio 31,6 mm (rango 17-46 mm); AAC promedio $128,95^{\circ}$ (rango $65-165^{\circ}$ ). En cuanto al tipo de terminación $41(95,3 \%)$ fueron simples, mientras que 2 casos $(4,7 \%)$ fueron de forma múltiple. Respecto a los afluentes, se encontraron en 42 de los casos (90,5\%). Se encontraron válvulas en 27 (62,8 \%) de los casos. La longitud promedio del AVC fue de 31,6 $\mathrm{mm}$, se dispuso en la mayoría de los casos en ángulo obtuso, presentando en la mayoría de los casos terminación de tipo simple. Además, en el 90,5\% se encontró afluentes que se agotaban en el AVC y en más de la mitad de los casos se encontraron válvulas. Se discuten las implicancias de estos hallazgos en la génesis de la disfunción de angioaccesos.
\end{abstract}

PALABRAS CLAVE: Anatomía; Vena; Cefálica; Arco; Variación.

\section{INTRODUCCION}

Las fístulas arteriovenosas (FAVs) nativas son menos propensas al desarrollo de trombosis e infección que la utilización de prótesis y catéteres y son, por tanto, el gold standard para la confección de accesos vasculares permanentes (Ravani et al., 2013). Sin embargo, la estenosis de las mismas constituye aún una problemática común, determinando la disfunción del angioacceso y en consecuencia una hemodiálisis ineficaz (Sigala et al., 2014; Sivananthan et al., 2014).

Las disfunciones de los angio-accesos del brazo se producen habitualmente por alteraciones en la salida venosa. Es sabido, que muchos sectores presentan una predisposición a la estenosis. El arco de la vena cefálica (AVC) juega un rol preponderante en dicha problemática, siendo uno de los sitios más frecuentes para el desarrollo de estenosis (Turmel-Rodrigues et al., 2000; Rajan et al., 2004).

La vena cefálica (VC) resulta de la reunión de las venas cefálica del antebrazo y mediana cefálica. Asciende por el margen lateral del músculo bíceps braquial, atraviesa la fascia clavipectoral en la extremidad inferior del triángulo deltopectoral y asciende a lo largo de este último, bajo la fascia o en un desdoblamiento de esta, hasta la proximidad de la clavícula (Fig. 1). Debajo de este hueso se incurva, formando el AVC, atraviesa la fascia clavipectoral y desemboca en la vena axilar (VA). En la mayor parte de los casos, las venas toraco-acromiales terminan en la VC, cerca de su desembocadura (Rouvière \& Delmas, 2005; Latarjet \& Ruiz Liard, 2011).

El AVC se define como la angulación final de dicha vena, antes de su abocamiento en el eje axilar (Kian \& Asif, 2008). Así mismo, en radiología, se describe como la porción central perpendicular de la $\mathrm{VC}$, en su recorrido a través del triángulo deltopectoral, para unirse a la VA (Rajan et al., 2003; Jaberi et al., 2007; Hammes et al., 2009).

La estenosis del AVC constituye la principal causa de disfunción de las fístulas braquicefálicas (Rajan et al., 2003; Hammes et al.; Sivananthan et al.; Turmel-Rodigues et al.).

La estenosis de dicho sector hemodinamicamente significativa tiene una incidencia que va del 30 al $77 \%$ dentro de las fístulas braquicefálicas disfuncionantes (TurmelRodrigues et al.; Rajan et al., 2003, 2004). 
De aquí la importancia de un conocimiento acabado de la Anatomía del AVC y sus principales variantes, objetivo del presente trabajo.

\section{MATERIAL Y MÉTODO}

Se utilizaron 43 regiones axilares de cadáveres adultos, 23 derechas y 20 izquierdas, de ambos sexos, fijados en solución en base a formaldehído, sin patologías ni abordajes previos de la región. Todas las disecciones fueron llevadas a cabo por los autores del trabajo en los laboratorios del Departamento de Anatomía, Facultad de Medicina, Universidad de la República, (Montevideo -Uruguay).

Se procedió al abordaje del triángulo deltopectoral y de la región axilar. Se realizó mediante una incisión paralela a la clavícula a $2 \mathrm{~cm}$ de la misma, desde el proceso coracoides coracoides hasta la extremidad medial del primer espacio intercostal, a $4 \mathrm{~cm}$ de la línea medio esternal. Luego, se continuó hacia abajo la incisión paralela a esta última y a $2 \mathrm{~cm}$ del reborde costal se incurvó hacia abajo y lateral paralela a este último hasta la línea medio axilar. A nivel braquial anterior se realizó incisión desde el proceso coracoides por la línea media anterior del brazo hasta la fosa cubital. Se rebatieron los planos superficiales y fascia superficial del músculo pectoral mayor. Luego se realizó la individualización y disección del surco deltopectoral y su contenido. Se separó de los haces directo y reflejo del músculo pectoral mayor. Se realizó la sección a $2 \mathrm{~cm}$ del húmero los haces de este último lo que permitió rebatirla medialmente. Finalmente, luego de su exposición y disección del AVC, de sus afluentes y de su termi-

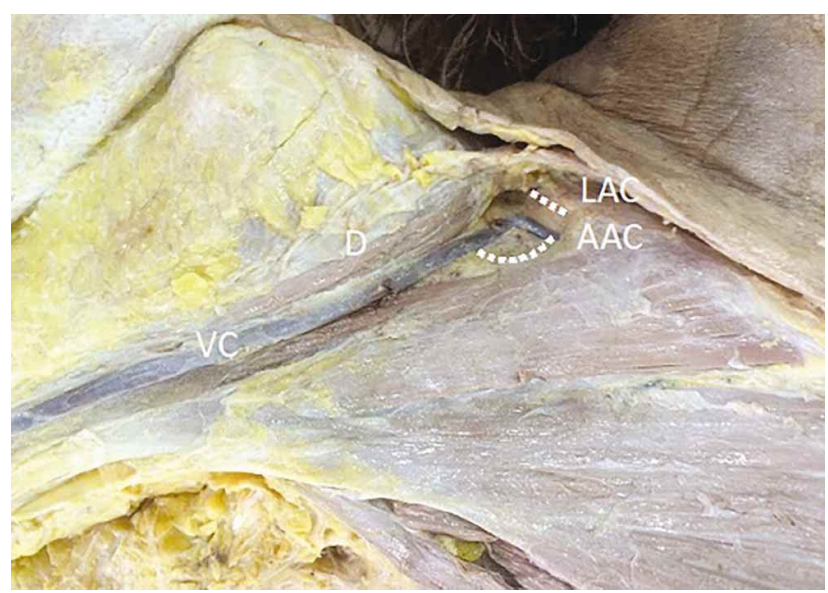

Fig. 1. Vista anterior del hombro derecho. Se observa el trayecto de la vena cefálica (VC) en el triángulo deltopectoral para alcanzar la vena axilar (VA). Se observan las medidas registradas: longitud del arco de la vena cefálica (LAC); ángulo del arco de la vena cefálica (AAC). Otras referencias: músculo deltoides (D); músculo pectoral mayor (PM). nación. Por último, se realizó una incisión longitudinal en la pared anterior del mismo, para la identificación de válvulas.

Se registraron las medidas con un calper milimetrado digital y semicírculo las siguientes mediciones y principales características anatómicas. Las mismas fueron tomadas con el cadáver en posición decúbito dorsal, con el miembro superior en abducción a 45 grados.

Se documentó la presencia del AVC y se registró: la longitud del AVC (LAC), la angulación del AVC respecto a la vena cefálica (AAC) (Fig. 1), el tipo de terminación (simple o múltiple), la presencia y número de afluentes; y por último, la presencia y número de válvulas.

\section{RESULTADOS}

En el $100 \%$ ( $\mathrm{n}=43$ ) de los casos se encontró la presencia del AVC, rodeado por la fascia clavipectoral, en su pasaje por el triángulo deltopectoral.

En cuanto a la morfología del AVC, se encontró un LAC promedio $31,6 \mathrm{~mm}$ (rango $17-46 \mathrm{~mm}$ ) y un AAC promedio fue de $128^{\circ} 6\left(\right.$ rango $\left.65-165^{\circ}\right)$.

En cuanto al tipo de terminación, se halló que el 95,3 $\%(\mathrm{n}=41)$ fueron simples, mientras que el 4,7 \% $(\mathrm{n}=2)$ tuvo una terminación de tipo doble. En ambos casos terminaron mediante 2 conductos separados, en la VA (Fig. 2).

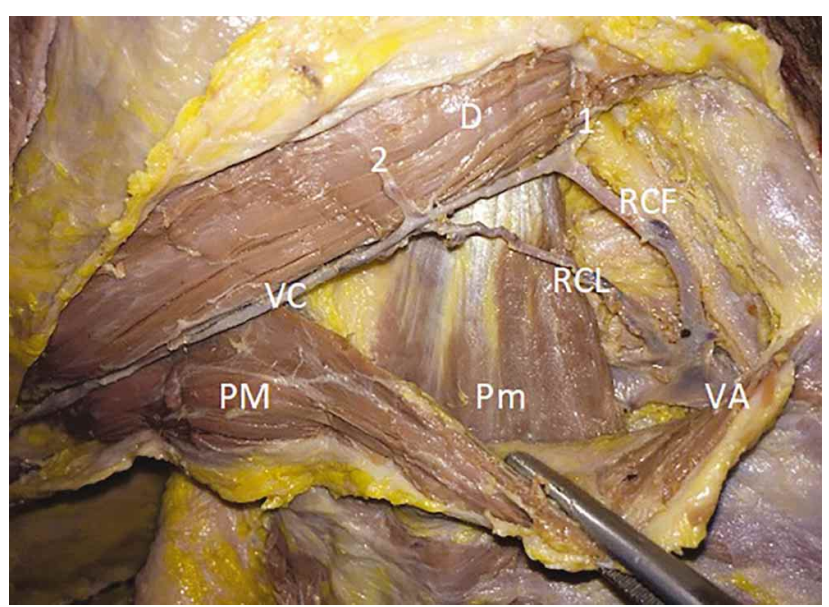

Fig. 2. Vista anterior del hombro derecho. Se observa la vena cefálica (VC) que a nivel del triángulo deltopectoral se bifurca en 2 ramas. La rama cefálica $(\mathrm{RCF})$ recibe a la vena tóraco-acromial (1) y la rama caudal (RCL) recibe una rama colateral deltoidea (2). Ambas desembocan en la vena axilar (VA). Se trata de un arco de la vena cefálica (AVC) bífido, con abocamiento en la VA. Otras referencias: músculo deltoides (D); músculo pectoral mayor (PM); músculo pectoral menor $(\mathrm{Pm})$. 
El 97,7\% (n=42) desembocó en la VA previo trayecto en el triángulo deltopectoral. Se encontró 1 caso $(2,3$ $\%$ ) de abocamiento en la vena yugular externa (VYE), luego de un trayecto supraclavicular, como un conducto único (Fig. 3).

Se encontraron afluentes en el $97,7 \%(n=41)$ de los casos, en un promedio de 2 por VC.

Respecto al número de válvulas, se registró la presencia de las mismas en el 62,8\% $(n=27)$, presentando cada caso un promedio de 1,4 válvulas.

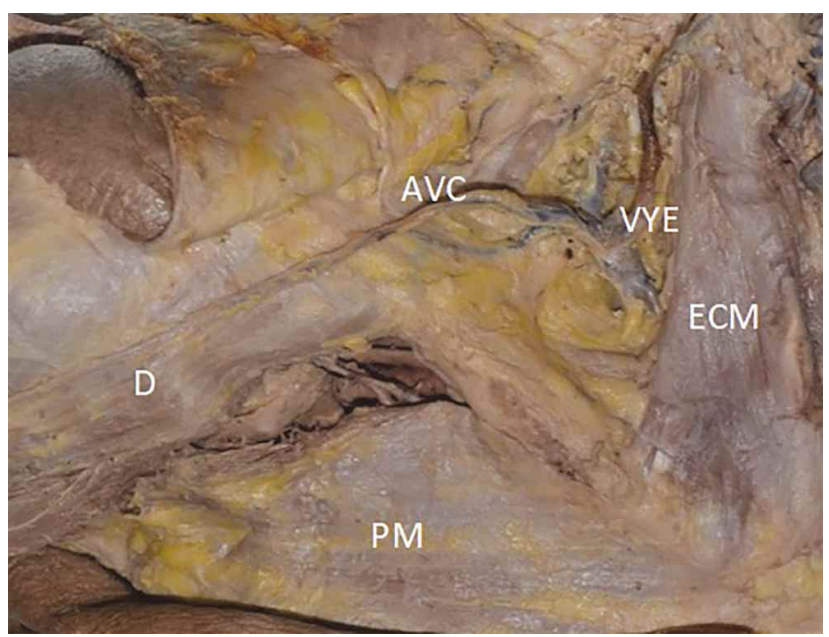

Fig. 3. Vista anterior del hombro derecho. La vena cefálica (VC) presenta un trayecto supraclavicular hasta alcanzar a la vena yugular externa (VYE), caudalmente al músculo esternocleidomastoideo (ECM). Se trata de arco de la vena cefálica (AVC) simple, con abocamiento en la VYE. Otras referencias: músculo deltoides (D); músculo pectoral mayor (PM); clavícula (C).

\section{DISCUSIÓN}

El desarrollo de estenosis en pacientes con FAVs braquiocefálicas presenta una predilección por el AVC (Hammes et al.; Rajan et al., 2003; Sivananthan et al.; Tummel-Rodrigues et al.). Se plantea, que el sitio más frecuente de localización es a nivel del sector terminal del AVC, es decir, en la terminación de la VC en la VA y disminuye en frecuencia cuanto más distal de la terminación nos encontremos (Bennett et al., 2015).

Las características anatómicas que juegan en el desarrollo de la estenosis venosa son: por un lado, la geometría del AVC. Por otro lado, el tipo de terminación, de acuerdo a si esta es simple doble o triple y así mismo, el sitio de abocamiento. También, la presencia de afluentes y de válvu- las. Se plantea, que estas causas podrían devenir en una eventual hiperplasia intimal, estrés endotelial y un mayor grado de flujo turbulento a dicho nivel (Iimura et al., 2003; Daoui \& Asif, 2012; Hammes et al.; Jaberi et al.; Sivananthan et al.).

Dentro de otras características a considerar, encontramos que la mayor parte del AVC se encuentra sometido a la compresión extrínseca que ejercen la tensión y el grosor de la fascia clavipectoral, así como también el músculo pectoral mayor. Se plantea, que estas estructuras no permitirían la dilatación apropiada del AVC frente a las demandas fisiológicas del estrés parietal, generando un aumento de la presión en dicho vaso, resultando en un incremento de la hiperplasia intimal e hipertrofia de las células musculares lisas (Daoui \& Asif; Iimura et al.; Sivananthan et al.).

Por otra parte, se agregan las alteraciones bioquímicas propias de la falla renal crónica, lo cual escapa al alcance de este trabajo (Daoui \& Asif).

La estenosis de la AVC puede generar una reducción progresiva del flujo, el desarrollo de trombosis y en algunos casos asociarse a una significativa dilatación preestenótica o aneurisma de la VC (Sivananthan et al.).

Las razones por las cuales el AVC presenta tendencia a la estenosis continúan en investigación. Como ya adelantamos, algunos autores han propuesto que se debe a características anatómicas propias del AVC y factores hemodinámicos, entre otras (Boghosian et al., 2014; Daoui \& Asif.; Hammes et al.; Iimura et al.; Jaberi et al.; Sigala et $a l$.; Sivananthan et al.). Nos centraremos fundamentalmente en los primeras.

En primer lugar, se encontró la presencia de un AVC en el $100 \%$ de los casos ( $\mathrm{n}=43)$, al igual que lo descrito por otros autores (Yeri et al., 2009).

En cuanto a la morfología del AVC, se encontró un LAC promedio $31.6 \mathrm{~mm}(17-46 \mathrm{~mm})$. La AAC promedio fue de $128^{\circ} 6 \notin$ (rango $65-165^{\circ}$ ). Los sitios de angulación se asocian frecuentemente al desarrollo de flujo turbulento, que estimula la proliferación endotelial, la hiperplasia intimal y la remodelación hipertrófica consecuente, vasoconstricción y agregación plaquetaria. Esto no solo se ha visto que incrementa la presión venosa transmural postfistula sino también el desarrollo de trombosis a dicho nivel (Daoui \& Asif.; Sivananthan et al).

Hammes et al. demostraron que pacientes diabéticos con FAVs braquicefálicas son menos propensos al desarrollo de estenosis de la VC en contraposición a pacientes no diabéticos. Esto puede deberse, a la geometría diferente del 
AVC en el grupo de diabéticos. Estos últimos, tienden a tener un ángulo mayor y un radio de curvatura mayor. Se plantea que el mayor flujo en paciente no diabéticos causa, en parte, la modificación en la geometría de estos pacientes (Hammes et al.).

Por otro lado, cabe destacar, que no se encuentran referencias respecto a la influencia del ángulo de abocamiento de la VC en la VA, como causante de estenosis a dicho nivel. Jaberi et al. demostraron una diferencia no significativa entre los ángulos de abocamiento de la VC en la VA entre pacientes con estenosis del AVC y paciente sanos (Jaberi et al.).

En cuanto al tipo de terminación, se encontró que el 95,3\% ( $\mathrm{n}=41)$ fueron simples, siendo ésta el tipo de terminación más frecuente, mientras que el 4,7 \% (n=2) tuvo una terminación de tipo doble.

De acuerdo a los estudios consultados, la terminación en forma simple es la conformación más frecuente, hecho concordante con nuestros resultados. Sin embargo, numerosos autores han señalado la existencia de terminaciones dobles del AVC, al igual que las encontradas en el presente estudio (Kian \& Asif, 2008; Daoui \& Asif; Russo et al., 2017; Maalman et al., 2018).

La terminación doble se denomina bifurcación o AVC bífido y sus ramas de terminación pueden terminar ambas en la VA o bien una de ellas en la VYE, como veremos a continuación (Kian \& Asif). Se han descrito también casos de trifurcaciones (Boghosian et al.).

Boghosian et al. compararon la terminación de la VC con registros hemodinámicos realizados mediante programas informáticos de dinámica de fluidos en pacientes con FAVs braquicefálicas, registrando mayor funcionalidad de las mismas en terminaciones múltiples, por lo cual este se trataría de un factor protector (Boghosian et al.). Con respecto al sitio de terminación, el 97,3\% $(n=41)$ desembocó a nivel de la VA, siendo el sitio de terminación más frecuente.

Existe variaciones anatómicas bien documentadas de la porción terminal de la VC (Lau et al., 2007). Las variaciones anatómicas de la VC en cuanto a su abocamiento son poco frecuentes, pero no despreciables (Loukas et al., 2008; Ghosal et al., 2014; Wysiadecki et al., 2016; Novakov \& Krasteva, 2018).

De los 2 casos de terminación doble que se encontraron, ambos desembocaron en la VA. En la literatura consultada, se encontraron casos similares a los nuestros, con terminación a nivel de la VA para ambas ramas de bifurcación.
También, casos de terminación de tipo doble pero con abocamiento independiente. En estos últimos, habitualmente, la rama superior drenaba en la VYE, con un trayecto previo supraclavicular, pasando por delante del tercio lateral de la clavícula. La rama inferior, de menor entidad, desembocaba en la VA. Dicha situación, no solo tiene implicancias a la hora de una cirugía vascular periférica, sino también en el contexto de traumatismos de clavícula, en otros, que escapan al alcance de este trabajo (Boghosian et al.; Kian \& Asif; Maalman et al.; Russo et al.; Yeri et al.).

En el presente trabajo se encontró un caso de abocamiento en la VYE. Esta variación tiene una frecuencia del 0,7\% al 5,4\% en la bibliografía consultada. Corresponde al remanente embriológico denominado vena yugulocefálica persistente (VYC), típica de primates no humanos, y reportada de manera esporádica en humanos (Jun et al., 2017; Latarjet \& Ruiz Liard; Maalman et al.; Novakov \& Krasteva; Russo et al.)

Así mismo, la literatura describe casos en los que el AVC puede drenar también en la vena yugular interna (VYI) o directamente en la vena subclavia (VS), siendo esta última, la variación más frecuente (Kiev \& Asif; Lau et al.).

Acerca de los afluentes, se encontraron en el 97,7\% $(\mathrm{n}=42)$ de los casos, siendo concordante con el expuesto por autores clásicos (Rouvière \& Delmas; Latarjet \& Ruiz Liard).

En cuanto a la presencia de válvulas, se registró su presencia en el 62,8\% $(\mathrm{n}=27)$, presentando cada caso un promedio de 1,4 válvulas. Esta cifra es similar a lo expuesto por Sivananthan y algo inferior a lo expuesto por Iimura (Iimura et al.; Sivananthan et al.).

La existencia de válvulas en el AVC ha sido descrita por varios autores, las cuales tienen por función prevenir el reflujo venoso a dicho nivel (Bennett et al.; Daoui \& Asif; Hammes et al.; Iimura et al.). El sector terminal del AVC es el sitio de localización más frecuente de las mismas, siendo estas el doble que en cualquier otro sector de la VC (Iimura et al. y Kiev \& Asif). Se describe un promedio de dos válvulas en el AVC en la mayoría de los individuos (Harmon \& Edwards, 1987) y se concentran en mayor parte en los 3 mm distales del mismo (Boghosian et al.; Daoui \& Asif; Kiev \& Asif; Lau et al.). La presencia de numerosas válvulas, especialmente en el sector más distal del AVC puede probablemente causar un mayor grado de flujo turbulento a dicho nivel, estrés endotelial produciendo hipertrofia intimal y reduciendo la luz del vaso significativamente, con la subsecuente estenosis. Esto posiblemente explique, en parte, la mayor prevalencia de estenosis en el sector terminal del AVC (Kiev \& Asif). 
Si bien no se trató del objetivo de nuestro estudio, se constató la presencia de la fascia clavipectoral en el $100 \%$ de los casos. Es importante señalar el concepto del remodelado vascular, el cual constituye la capacidad de la vena de dilatarse en presencia de flujo de alto grado a través de la fístula. La capacidad del AVC de remodelado y dilatación de su luz podría estar limitado por la presencia y las características de la fascia clavipectoral, la cual es una densa membrana fascial a través de la cual el AVC debe transcurrir para alcanzar la VA. La presencia de la fascia clavipectoral y la tensión que ejerce sobre el AVC, podría impedir que este alcance no solo la remodelación sino también la angulación necesaria, para un flujo venoso óptimo (Rajan et al., 2003).

Así mismo, la VC no sería capaz de dilatarse de acuerdo al grado de flujo por lo que es muy probable que se transforme en turbulento, especialmente en sitios como este donde el flujo es de por sí alto, deviniendo en daño e hiperplasia endotelial (Jaberi et al.).

Es así que la presencia de válvulas, la fascia clavipectoral, los músculos pectoral mayor, pectoral menor y deltoides, así como la movilidad resultante en el triángulo deltopectoral parecen en algunos casos contribuir al desarrollo de estenosis (Daoui \& Asif; Harmon \& Edwards; Kiev \& Asif; Yeri et al.).

\section{CONCLUSIONES}

En el presente trabajo se encontraron ciertas variantes en la morfología, en la presencia de válvulas, en el tipo de abocamiento, así como en el sitio de terminación del AC. De la revisión bibliográfica surge que las variaciones en las características anatómicas de la AVC en su longitud, angulación, afluentes y forma de abocamiento podrían estar relacionadas en la génesis de la estenosis del AVC y en la predilección por su sector terminal de dicha complicación. Es por todo esto que jerarquizamos la importancia del conocimiento anatómico detallado del AVC en vistas a la planificación de la confección de los angioaccesos y al eventual tratamiento de las disfunciones de los mismos.

MANSILLA, A.; MANSILLA, S.; POUY, A.; GARRETANO, A.; AMÉNDOLA, J. I.; RUSSO, A. \& OLIVERA, E. Cadaveric study of the cephalic vein arch. Int. J. Morphol., 38(5):1229-1234, 2020.

SUMMARY: Within the angio access dysfunctions, the stenosis of the cephalic vein arch (AC) has a significant role. There are anatomical characteristics of AVC that partially explain this phenomenon. This requires a deep understanding of AVC and its main variants. 43 axillary regions of formalin-fixed adult cadavers were dissected. For this study, the following were registered: length of the AVC (LAC), angulation of the AVC with respect to the cephalic vein (AAC), type of termination (single or multiple), presence and number of tributaries. LAC average $31.6 \mathrm{~mm}$ (range $17-46 \mathrm{~mm}$ ); AAC average $128,95^{\circ}$ (range $65-165^{\circ}$ ). Regarding the type of termination, $41(95.3 \%)$ were simple, while 2 cases (4.7 $\%)$ were multiple. Tributaries were found in 42 of the cases $(90.5$ $\%)$. Valves were found in $27(62.8 \%)$ of the cases. The average length of the AVC was $31.6 \mathrm{~mm}$. Most cases presented an obtuse angle, and simple termination. In addition, in $90.5 \%$ tributaries were found and in more than half of the cases valves were found. Implications of these findings in the genesis of angioaccess dysfunction are discussed.

KEY WORDS: Anatomy; Vein cephalic; Arch; Variation.

\section{REFERENCIAS BIBLIOGRÁFICAS}

Bennett, S.; Hammes, M. S.; Blicharski, T.; Watson, S. \& Funaki, B. Characterization of the cephalic arch and location of stenosis. J. Vasc. Access, 16(1):13-8, 2015.

Boghosian, M.; Cassel, K.; Hammes, M.; Funaki, B.; Kim, S.; Qian, X.; Wang, X.; Dhar, P. \& Hines, J. Hemodynamics in the cephalic arch of a brachiocephalic fistula. Med. Eng. Phys., 36(7):822-30, 2014.

Daoui, R. \& Asif, A. Cephalic arch stenosis: mechanisms and management strategies. Semin. Nephrol., 32(6):538-44, 2012.

Ghosal, T.; Begum, S.; Roy, T. \& Gupta, I. A rare variation of superficial venous drainage pattern of neck. Int. J. Anat. Radiol. Surg., 3(4):1-3, 2014.

Hammes, M. S.; Boghosian, M. E.; Cassel, K. W.; Funaki, B. \& Coe, F. L. Characteristic differences in cephalic arch geometry for diabetic and non-diabetic ESRD patients. Nephrol. Dial. Transplant., 24(7):2190-4, 2009.

Harmon, J. V. Jr. \& Edwards, W. D. Venous valves in subclavian and internal jugular veins. Frequency, position, and structure in 100 autopsy cases. Am. J. Cardiovasc. Pathol., 1(1):51-4, 1987.

Iimura, A.; Nakamura, Y. \& Itoh, M. Anatomical study of distribution of valves of the cutaneous veins of adult's limbs. Ann. Anat., 185(1):91$5,2003$.

Jaberi, A.; Schwartz, D.; Marticorena, R.; Dacouris, N.; Prabhudesai, V.; McFarlane, P. \& Donnely, S. Risk factors for the development of cephalic arch stenosis. J. Vasc. Access, 8(4):287-95, 2007.

Jun, E. S. W.; Lun, A. L Y. \& Nikam, M. A rare anatomic variant of a single-conduit supraclavicular cephalic arch draining into the external jugular vein presenting with recurrent arteriovenous fistula stenosis in a hemodialysis patient. J. Vasc. Surg. Cases Innov. Tech., 3(1):20$2,2017$.

Kian, K. \& Asif, A. Cephalic arch stenosis. Semin. Dial., 21(1):78-82, 2008.

Latarjet, M. \& Ruiz Liard, A. Anatomía Humana. $4^{\mathrm{a}}$ ed. Buenos Aires, Médica Panamericana, 2011.

Lau, E. W.; Liew, R. \& Harris, S. An unusual case of the cephalic vein with a supraclavicular course. Pacing Clin. Electrophysiol., 30(5):719-20, 2007.

Loukas, M.; Myers, C. S.; Wartmann, Ch. T.; Tubbs, R. S.; Judge, T.; Curry, B. \& Jordan, R. The clinical anatomy of the cephalic vein in the deltopectoral triangle. Folia Morphol. (Warsz.), 67(1):72-7, 2008. 
Maalman, R. S. E.; Donkor, Y. O.; Ayamba, A. M. \& Abledu, J. K. A rare anatomical variation of the termination of right and left cephalic veins. Case Rep. Vasc. Med., 2018:5809656, 2018.

Novakov, S. \& Krasteva, E. Anatomical variants in the termination of the cephalic vein. Acta Morphol. Anthropol., 25(3-4):132-8, 2018.

Rajan, D. K.; Bunston, S.; Misra, S.; Pinto, R. \& Lok, C. E. Dysfunctional autogenous hemodialysis fistulas: outcomes after angioplasty--are there clinical predictors of patency? Radiology, 232(2):508-15, 2004.

Rajan, D. K.; Clark, T. W.; Patel, N. K.; Stavropoulos, S. W. \& Simons, M. E. Prevalence and treatment of cephalic arch stenosis in dysfunctional autogenous hemodialysis fistulas. J. Vasc. Interv. Radiol., 14(5):56773, 2003.

Ravani, P.; Palmer, S. C.; Oliver, M. J.; Quinn, R. R.; MacRae, J. M.; Tai, D. J.; Pannu, N. I.; Thomas, C.; Hemmelgarn, B. R.; Craig, J. C.; et al. Associations between hemodialysis access type and clinical outcomes: a systematic review. J. Am. Soc. Nephrol., 24(3):465-73, 2013.

Rouvière, H. \& Delmas, A. Anatomía Humana. 11ª ed. Barcelona, Masson, 2005.

Russo, A.; Cubas, S.; Mansilla, A.; Mansilla, S. \& Olivera, E. Variants of the cephalic arch: report of 2 cases. Int. J. Anat. Var., 10(3):64-5, 2017.

Sigala, F.; Saßen, R.; Kontis, E.; Kiefhaber, L.; Förster, R. \& Mickley, V. Surgical treatment of cephalic arch stenosis by central transposition of the cephalic vein. J. Vasc. Access, 15(4):272-7, 2014.

Sivananthan, G.; Menashe, L. \& Halin, N. J. Cephalic arch stenosis in dialysis patients: review of clinical relevance, anatomy, current theories on etiology and management. J. Vasc. Access, 15(3):157-62, 2014.

Turmel-Rodrigues, L.; Pengloan, J.; Baudin, S.; Testou, D.; Abaza, M.; Dahdah, G.; Mouton, A. \& Blanchard, D. Treatment of stenosis and thrombosis in haemodialysis fistulas and grafts by interventional radiology. Nephrol. Dial. Transplant., 15(12):2029-36, 2000.

Wysiadecki, G.; Polguj, M. \& Topol, M. Persistent jugulocephalic vein: case report including commentaries on distribution of valves, blood flow direction and embryology. Folia Morphol. (Warsz.), 75(2):271-4, 2016.

Yeri, L. A.; Houghton, E. J.; Palmieri, B.; Flores, M.; Gergely, M. \& Gómez, J. E. Cephalic vein. Detail of its anatomy in the deltopectoral triangle. Int. J. Morphol., 27(4):1037-42, 2009.

\section{Corresponding author: \\ Dr. Alejandro Russo \\ Caning 2505/306 \\ CP 11300 \\ Montevideo \\ URUGUAY}

Email: aleru86@gmail.com

Received: 23-02-2020

Accepted: 27-04-2020 